\title{
Collection and Transmission System of Radiation Information With a Portable Radiation Detector
}

\author{
Takanori NAGATANI $^{\text {a, }}{ }^{*}$, Hideaki KATAYAMA ${ }^{\text {a }}$, Shigeyasu NAKAGAWA ${ }^{\text {a }}$ \\ ${ }^{a}$ Dept. of Electrical and computer engineering, National Institute of Technology, Maizuru College, \\ 234 Shiraya, Kyoto 625-8511, Japan \\ *Corresponding Author: a0339@g.maizuru-ct.ac.jp,
}

\begin{abstract}
The necessity of radiation measurement has been increasing since the accident at the Fukushima nuclear power plant. But general radiation detector is very expensive. So it is difficult to increase the number of radiation monitoring stations. On the other hand, inexpensive radiation measuring instruments have been sold for private use. So our purpose is to develop the system that enables us to collect and transmit radiation information with using a portable radiation detector. Then we developed sensor network to collect radiation information using a portable radiation detector. Power consumption of sensor nodes were reduced about $40 \%$ by sleep function. We confirmed operation with four sensor nodes. We developed web application that helps to collect and transmit radiation information of both wireless sensor network and radiation monitoring stations operating in Maizuru.
\end{abstract}

Keywords: Radiation, wireless sensor network web application.

\section{Introduction}

Since the Fukushima nuclear accident in the Great East Japan Earthquake of March 2011, there has been a growing interest in the radiation of personal belongings. At the same time, there has been an increasing demand in the information of Spatial Distribution of radiation dose in the region and continuous measurement ${ }^{(1)(2)}$. Moreover, a public institution such as Nuclear Regulatory Agency have transmitted observation results of radiation dose by its' own website.

There are seven radiation monitoring stations at work in Maizuru, uploading radiation information onto the Internet. In contrast, there are 479 radiation monitoring stations at work in Iwaki, Fukushima Prefecture. Seven monitoring stations are insufficient in number to grasp spatial distribution of radiation in Maizuru. One of the reasons why there are not enough monitoring stations in Maizuru is that instruments for measuring the spatial distribution of radiation is generally expensive, and that it is costly to install radiation monitoring stations. These days, we can buy a reasonable radiation detector and radiation detector kits for private use. We are able to measure radiation dose by myself. In addition, we are able to upload measured radiation dose to website. However, it have never reported a system that continuously collect radiation dose and upload collected information to website.

The purpose of this study was to develop the system that continuously collect radiation information and transmit collected information. Therefore, we develop the sensor network using a portable radiation detector. And we develop the sleep function for power reduction. Then we develop a system that collect and transmit the radiation information by developing sensor network through wireless communication.

\section{System Configuration}

This system is divided into sensor network section and gathering transmission section for radiation information. Sensor network section measures radiation dose and send radiation dose measured through wireless communication. Collection transmission unit collects, stores and transmits measured radiation dose. Fig. 1 shows configuration diagram of the system that we developed.

\subsection{Constitution of sensor network}

Sensor network is composed of sensor nodes, a coordinator and repeaters. Sensor nodes measure radiation 
dose. Sensor node send result of measurement to the coordinator. The Coordinator inputs the received result of measurement into computer. The repeater relays radiation information by wireless communication.

\subsection{Constitution of radiation information collection transmitting unit}

Radiation information collection transmitting unit is composed of the server and Raspberry PI. The server functions to collect and store radiation information. Stored radiation information is transmitted to local residents by web application. The Server is composed of CentOS, Apache, MySQL and PHP.

Raspberry PI sends radiation information received by sensor network to the server. Raspberry PI's OS is Linux. So that it can be used like a server computer. Besides, it is easy to connect Raspberry PI to the network ${ }^{(3)}$. Therefore, we have decided to use Raspberry PI for the system.

\section{Sensor Network}

\subsection{An overview of the wireless sensor network}

We adopt multi-hop communication for the sensor network of the system. The features of the multi-hop communication are as follows:

- We can arrange sensor nodes freely because we don't have to lay a cable.

- When we add a sensor node, the network is renewed automatically.

- Even if a sensor node fail or the battery runs out, sensor network can continue to gather information, because the another sensor node is connected automatically ${ }^{(4)}$.

A problem for the wireless sensor network is how to reduce power consumption of the sensor node. We adopts that the sensor node is became this problem. When sensor node does not make a measurement, it waits in sleep mode. So, power consumption is reduced. In sleep mode, sensor node cannot accept a signal from other devices. So it automatically recovers from sleep mode, makes measurements, and sends the result to coordinator.

\subsection{Components}

A sensor node consists of an AIRCOUNTER, a Xbee and an Arduino. Fig. 2 shows the components of the wireless sensor network.

AIRCOUNTER is a portable radiation detector sold by S.T.CORPORATION. AIRCOUNTER is a kind of semiconductor detector. It is capable of UART connection by RS232C. Radiation detector employed UART connection is unusual. AIRCOUNTER is inexpensive and easy to control. For example, start and measures radiation. For these reasons, we have adopted AIRCOUNTER ${ }^{(5)(6)}$.

$\mathrm{XBee}$ is a wireless communication module sold by Digi International. It conforms to IEE802.15.4 and ZigBee standards, and so is ideal for constructing a wireless sensor network. In addition, it has sleep mode ${ }^{(7)}$.

Arduino is a Microcomputer board. It has a library for XBee control and serial communication. So it the best qualified to make a prototype ${ }^{(8)}$.

The coordinator and repeaters, which do not measure, are composed of an Arduino and a XBee.

\section{Collection transmission section} for radiation information

\section{Wireless sensor network}

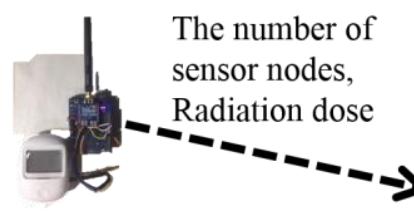

adiation information from

radiation monitoring stations

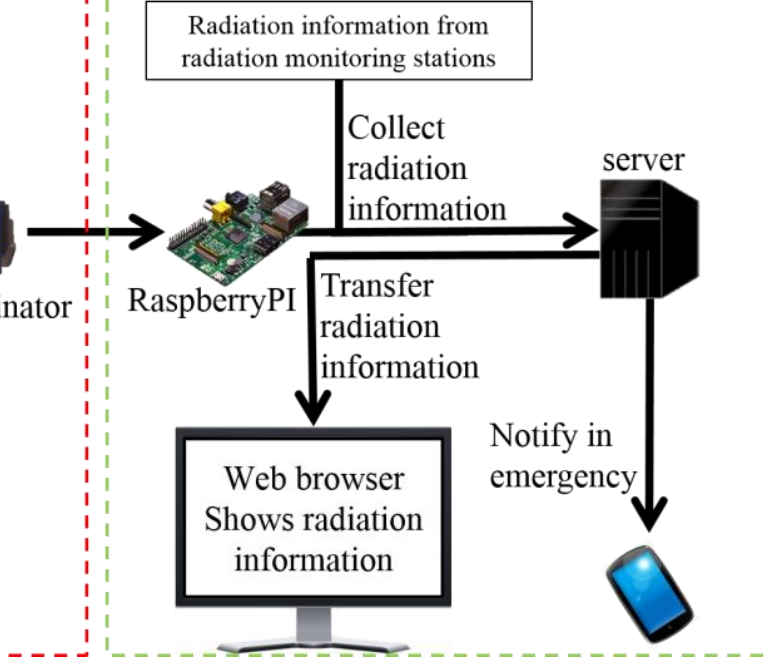

Fig.1. Configuration diagram of system 


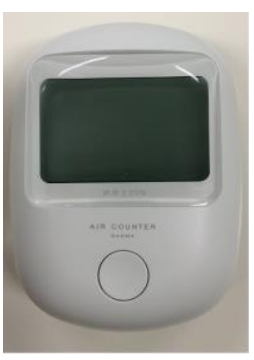

AIRCOUNTER

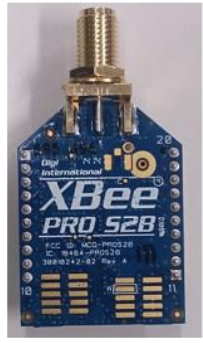

XBee

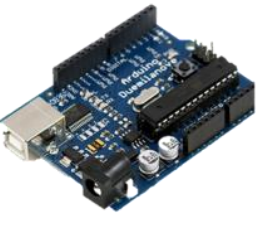

Arduino
Fig. 2. Components of the wireless sensor network.

\subsection{Operation of sensor node}

We explain the operation of the sensor node in this section.

Fig.3 shows the cycle which sleep mode and measurement of sensor node. When the sensor node is in the sleep mode, it uses watchdog timer in Arduino. The sensor node releases the sleep mode of microcomputer board and XBee when watchdog timer finishes counting. Then the sensor node resumes measurement. The sensor node switches to the sleep mode when it recognizes that the result of measurement has been sent to the coordinator.

Fig.4 shows the communication process of AIRCOUNTER, Arduino and XBee. First, Arduino commands AIRCOUNTER to start and make measurements. On receiving the command, AIRCOUNTER starts to measure. AIRCOUNTER returns a measured value to Arduino. At this time, AIRCOUNTER passes to Arduino the measured dose in the unit called CPS. CPS represents the frequency of gamma rays detected per second ${ }^{(9)}$. Arduino converts CPS to $\mathrm{Sv} / \mathrm{h}$. Sv/h is a unit which shows the effect of radiation on the human bo $\mathrm{dy}^{(9)}$. Arduino gives measured dose to XBee when measurement is finished, and XBee sends measurement result to the coordinator. In addition to the measurement result, sensor nodes send XBee address to coordinator. By so doing, the coordinator identifies sensor nodes.

\subsection{Operation of coordinator}

The coordinator receives the radiation dose measured by sensor nodes with the use of XBee. Received radiation dose is sent to computer connected by USB. Coordinator sends radiation dose and XBee address. Xbee address is used to identify the sensor node. Serial communication is used for the communication of information.

\section{Information Gathering Transfer Section}

We would like to compare assembled data. In order to do so, we need radiation information of other areas. This system stores radiation information in the server. The server collects radiation information of wireless sensor network and radiation monitoring stations in Maizuru. Collected information of radiation is stored in the database. The server transmits radiation information to local residents.

The server also posts radiation information for local residents if sensor network detects high radiation value.

\subsection{Collection information with sensor network}

Radiation information on the sensor network is sent to the server through Raspberry PI. Sensor node sends measurement result at regular intervals. Sensor network leaves the timing of transmission to sensor node. RaspberryPI stores received radiation value. RaspberryPI calculates the average of the stored measurement values in 10 minutes and sends it every 10 minutes to the server, where it is stored in the database.

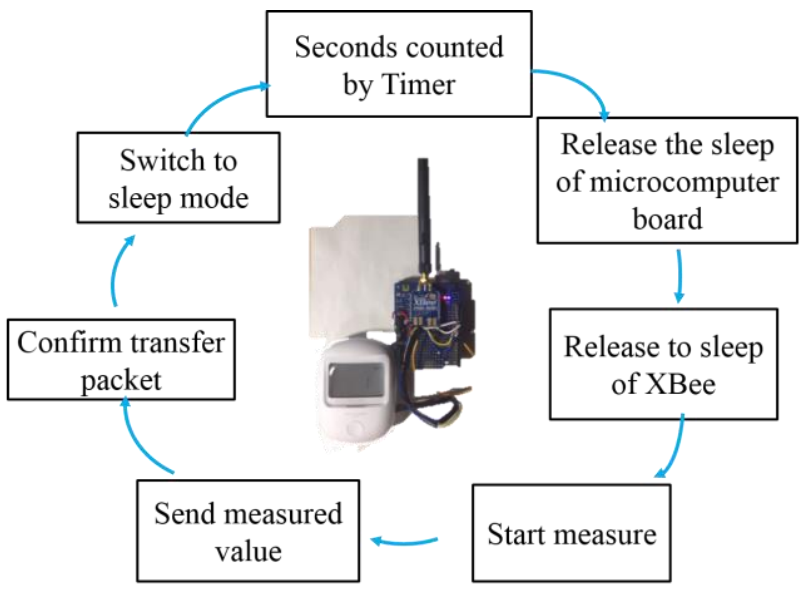

Fig. 3. Operation of sensor node.

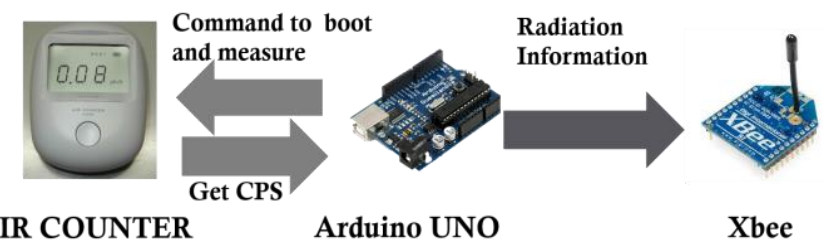

Fig. 4. Communication process of AIRCOUNTER, XBee and Arduino. 


\subsection{Collection information with radiation monitoring stations}

Information of radiation measured at radiation measurement stations in Maizuru is disclosed on a web page called "Disaster environment $\mathrm{N}$ net" ${ }^{(10)}$. The server of our system obtains radiation information from this web page. Radiation is measured every ten minutes in measurement stations. However, there is no fixed time for the measurements to be updated. The server accesses "Disaster environment $\mathrm{N}$ net" regularly, gains radiation information and loads it into the data base if this web page has been updated.

\subsection{Sending out information}

Radiation information stored in the database is sent out through web application. Radiation information is shown graphically and on a map. A graph tells us the change of radiation dose in terms of time. A map helps us to confirm radiation value in the whole region.

\subsection{Emergency notice of radiation information}

We should escape from radiation source if the sensor node detects a high dose of radiation. So we need a mechanism which functions to communicate radiation information to us in case of emergency. Since it is too late for escape when sensor nodes detect high levels of radiation, it sends out information when radiation value is gradually increasing. AIRCOUNTER often return result included error, and so the radiation information is not posted until more than one sensor node detects high radiation doses.

\section{Prototype}

\subsection{The prototype of sensor network}

Fig.5 shows the prototype of sensor network. Fig.6 shows the result of the received radiation information. This figure shows radiation information sent to computer from coordinator in JSON format. "FromSH" in the Fig.6 is the upper address of XBee. Because the upper address of XBee is the same value, it cannot be used for the identification of sensor nodes. On the other hand, each XBee has a different "SL", the lower address of Xbee, and so sensor nodes use this lower address for identification. "Ct" shows measurement time in units of seconds. "Svh" shows radiation value measured in units of $\mathrm{nSv} / \mathrm{h}$. "St" shows the state of each sensor node. Coordinator sends data to
Raspberry PI when "St" is "Stating". We have confirmed that up to four sensor nodes can be operated.

\subsection{Measurement of power consumed by sensor nodes}

Developed sensor nodes were reduced power consumption by sleep function. We would like to verify effectiveness of power reduction with sleep mode.

Moreover, we would like to found a component that is the highest power consumption. Therefore, we measured power consumption of sensor nodes. We measured current of a sensor node so that we measured power. Fig.7 shows the circuit for measuring the electric current. We measure the current at points A and B. Point A measures current of a whole sensor node. Point B measures current of AIRCOUNTER. Input voltage of sensor nodes is 9V. Table1 shows the experimental results. This result shows that power of sleep mode is about $40 \%$ smaller than sensor nodes of idol mode. Sensor nodes were able to stop to supply power to AIRCOUNTER if sensor node is sleep mode. Moreover, cause of power consumption is Arduino. Because, current of AIRCOUNTER is 0.006A. This current is smaller than a whole sensor node. Current of XBee is $15 \mathrm{~mA}$ if sensor nodes are idle mode. Current of $\mathrm{XBee}$ is $3.5 \mu \mathrm{A}$ if sensor nodes are idol mode ${ }^{(8)}$. Therefore, we should reconsider microcomputer board for reduction of power consumption

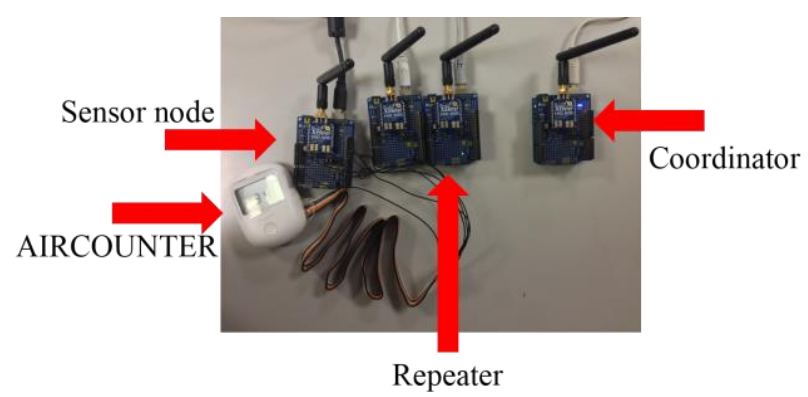

Fig. 5. The prototype(Sensor node,pepeater,coordinator).

["f romSH":"0013a200", "SL":"40a5620f", "ct": 120 ,"Svh": 51 ,"st":"Start ing $\left.{ }^{\prime \prime}\right\}$

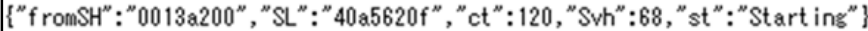
["f romSH":"0013a200", "SL":" 40a5620f", "ct" $: 120$, "Svh" $: 34$, "st": "Starting" \}

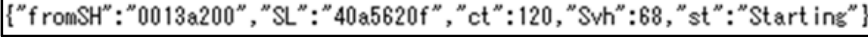

Fig.6. Information sent by coordinator

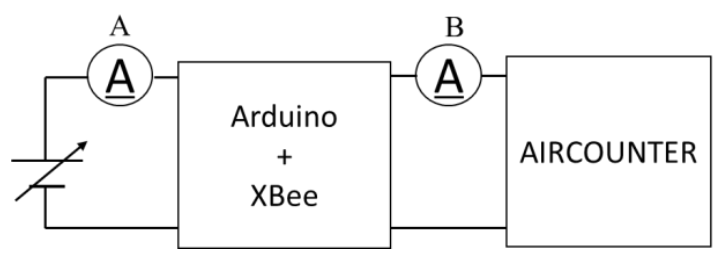

Fig.7. measured electric circuit 
Table 1. Measured current at sensor node.

\begin{tabular}{|c|c|c|}
\hline $\begin{array}{c}\text { Measurement } \\
\text { points }\end{array}$ & $\begin{array}{c}\text { During sleep } \\
\text { mode[A] }\end{array}$ & $\begin{array}{c}\text { During } \\
\text { measurement[A] }\end{array}$ \\
\hline $\mathrm{a}$ & 0.45 & 0.080 \\
\hline $\mathrm{b}$ & 0 & 0.006 \\
\hline
\end{tabular}

Table 2. Current at sensor node at point $A$ if input voltage change.

\begin{tabular}{|c|c|c|}
\hline $\begin{array}{c}\text { input } \\
\text { voltage[V] }\end{array}$ & $\begin{array}{c}\text { During sleep } \\
\text { mode[A] }\end{array}$ & $\begin{array}{c}\text { During } \\
\text { measurement[A] }\end{array}$ \\
\hline $5.0[\mathrm{~V}]$ & 0.02 & 0.08 \\
\hline $9.0[\mathrm{~V}]$ & 0.05 & 0.07 \\
\hline $12.0[\mathrm{~V}]$ & 0.05 & 0.07 \\
\hline
\end{tabular}

We verify to change performance of sleep mode by input voltage. Arduino is able to get power from DC power plug and USB port. If Arduino gets power from DC power plug, input voltage range of Arduino is values between 7 and $12 \mathrm{~V}$. If Arduino gets power from USB port, input supply voltage is $5 \mathrm{~V}$. We measured current at point $\mathrm{A}$ if input voltage is 5, 9 and $12 \mathrm{~V}$. Table 2 shows experimental result. From this result, power of sleep mode is about $30 \%$ smaller than idol mode if we use any input supply voltage.

These experiments shows that Arduino uses most of power consumption is Arduino. Therefore, we should change Arduino to a microcomputer such as PIC to reduce power consumption of sensor nodes. Moreover, power consumption of a sensor node don't change if input voltage change. We should focus on current capacity to select battery as far as voltage of battery is within the input voltage range of Arduino.

\section{Web Application}

In this section, we explain web application to confirm radiation information. Fig.8 shows top page of the web application. Radiation information of the whole region is shown on the map. We can select information at a certain time and a place with the selection box circled in red. Fig.8 shows graph by selected time. This graph shows radiation dose in transition from 9am to 12am on January $9^{\text {th }}, 2015$. This graph shows radiation information obtained through the sensor network and from radiation measurement stations running in Maizuru. Clicking icon on the map, we can display the information window as shown in Fig.10. The information window shows point name and radiation dose measured there. Clicking on a link calls up the graph in
Fig.11. This graph shows the transition of radiation dose current on the previous day.

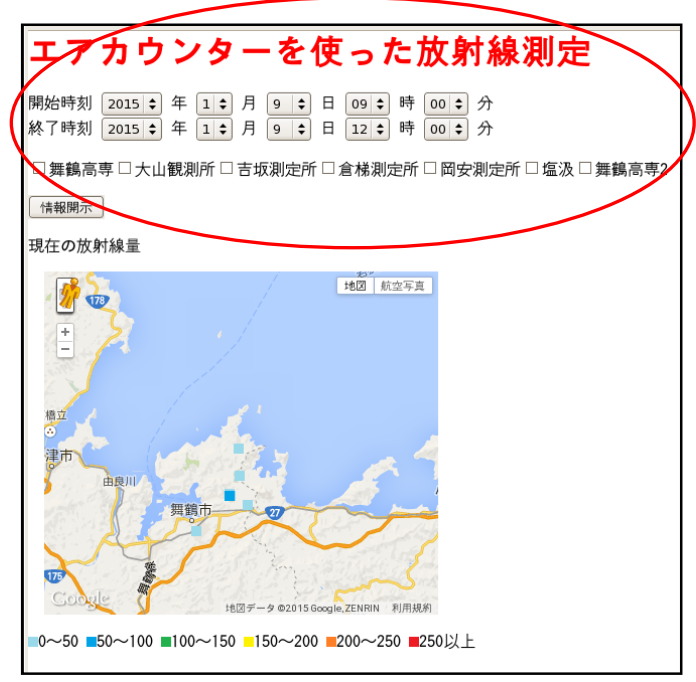

Fig. 8. Top page of web application.

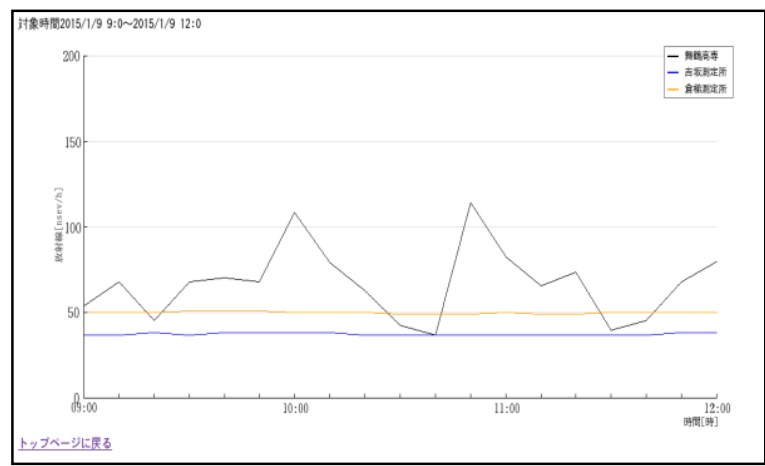

Fig.9. Graph display on web application.

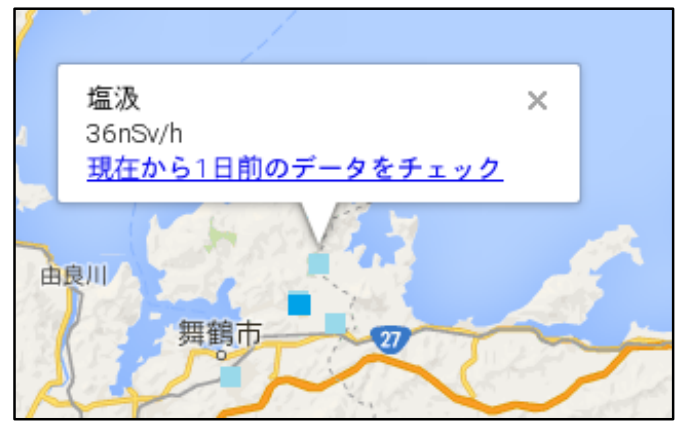

Fig. 10. Information window

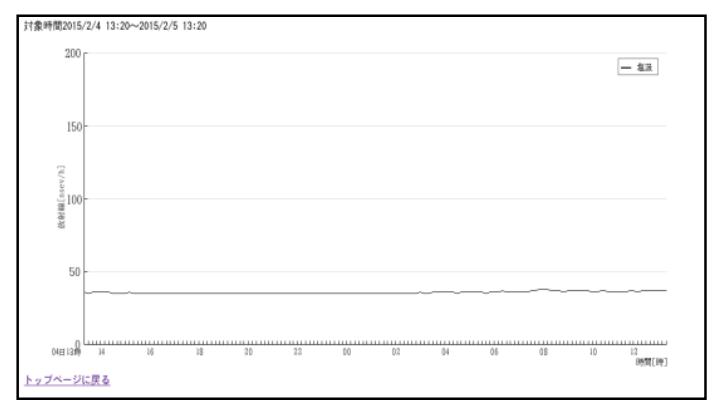

Fig.11. Graph shown by the link of information window 


\section{Checking the operation of emergency notification function}

We have checked the function of emergency notification. We prepared a lantern wick as a radiation source. We put sensor node close to it. By so doing, we heightened detected radiation dose. If sensor node detects more than $100 \mathrm{nSv} / \mathrm{h}$ of radiation dose, it is considered to have detected a high dose of radiation. In our experiment, because a small number of sensor nodes have been made by the prototype, the server is supposed to send an e-mail to the users if two sensor nodes detect a high radiation dose. Fig. 12 shows an e-mail sent to the users. The name and radiation dose of the sensor node are written in this e-mail. This time, the server posted information for the users when a detected radiation dose was above the threshold. If we set conditions, however, we think that it can notify the state of radiation rising gradually.

\section{Summary}

In this study, we have developed the system that functions to collect and transmit radiation information.

We have developed a wireless sensor network to measure radiation by using a reasonable radiation detector, an Arduino and a XBee. We confirm to operate wireless sensor network by 4 sensor nodes. Power consumption of sensor nodes were reduced by sleep function.

We developed a system that collect and transmit radiation information from wireless sensor network and radiation measurement stations in Maizuru. We were able to confirm to stably collect data from wireless sensor network. We were able to stably collect data from radiation measurement stations in Maizuru. If this system confirm to convert radiation data values, it have become to notify convert radiation data.

We have two pints of improvement in the system. First, we should change microcomputer board of sensor node from Arduino to microcomputer. By changing the parts, we reduce

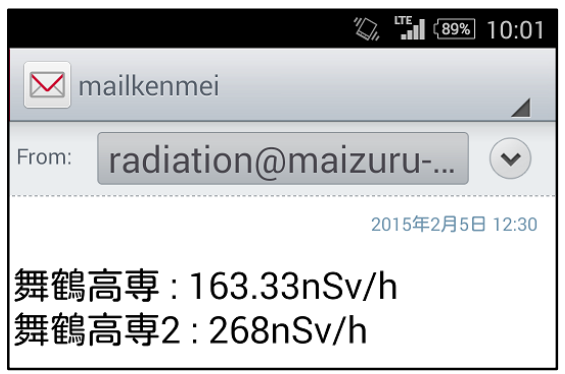

Fig. 12. An example of the experiment mail. power consumption of sensor nodes. Additionally, we can make a sensor node at low price.

Second, we should disclose information on evacuation. Local residents need to know where they can evacuate. This can be solved by pasting URL on the email for emergency notice. By using this URL, they will be able to access the server and draw information on shelters.

\section{References}

(1) Masaki Yamamoto : Changing markets for radiation measuring instrument industry since the great earthquake: Can this be a new business area after the earthquake?, Current analysis Report, Vol. 4, No. 96, pp. 1-2,2012

(2) National Institute of Science and Technology Policy : "The Change of the Public Attitudes to Science and Technology - - The Findings from Face-to-Face Interviews and from a Monthly Internet Survey-“, Research Document of Ministry of Education, No211,pp.110-124,2012

(3) Japanese Raspberry Pi Users Group, M.Ota H.Tyonan A.Ouchi Y.Otsuka H.Aoshima Y.Ikeda N.Kabaya K.Komura : "Be initiated into RaspberryPi”, Gijutsu-Hyohron Co., Ltd, pp5-10, 2013

(4) L.Zheng: "Zigbee Wireless Sensor Networks", journal of IEIE, vol.31, NO11, pp.854-857, 2011

(5) S.T Co: “AIRCOUNTER" (Access August 2014) http://www.st-c.co.jp/air-counter/

(6) S.Oohashi : "Peformance evalution of AIRCOUNTER that is household radiation detector, master's thesis of Tokyo Metropolitan University, 2012

(7) A.Watanabe : "an elementary knowledge of XBee that standard wireless communication module" , CQ Publishing Co., Ltd, Transistor technology, Vol49, No12, pp98-104, 2012

(8) Y.Kanzaki : Various measuring techniques in Arduino, CQ Publishing Co., Ltd, pp9-16,71,164, 2012

(9) E.Yosihda K,Kusama : "To master a radiation detector”, Journal of Home Economics ,Vol63 No.3, pp43-44, 2012

(10) Nuclear Regulation Authority :

Disaster environment N net, (Access March 2015) http://www.bousai.ne.jp/vis/index.php 